\title{
Dietmar Hansch
}

\section{Verfahrensintegrierende Verhaltenstherapie bei Angsterkrankungen -Lernen und Verlernen von pathologischer Angst als ganzheitlicher Prozess}

\section{Die Zersplitterung der Verhaltenstherapie - Lernen als ganzheitlicher Prozess}

Die Behandlung von Angsterkrankungen gilt mit Recht als eine der Paradedisziplinen der Verhaltenstherapie. Sowohl die kognitiven als auch die konfrontativen Verfahren zeigen seit Jahrzehnten in qualitativ hochwertigen Studien mittlere bis hohe Effektstärken.

Vom reinen Wirksamkeitsnachweis verschiebt sich inzwischen das Forschungsinteresse mehr in Richtung der Frage nach den entscheidenden Wirkfaktoren und einer Optimierung der Verfahren (Hoyer \& Heinig 2015). Vieldiskutiert ist derzeit das Konzept des Inhibitionslernens, nach dem sich pathologische Furchtassoziationen nicht auflösen, sondern durch neu gelernte Strukturen gehemmt und überlagert werden, die für ein gesundes Bewältigungsverhalten stehen. Untersucht wird derzeit, welche Faktoren und Strategien bei einer Expositionstherapie das Inhibitionslernen besonders fördern (Craske et al.,2014). Der hier vorgestellte Ansatz ist mit diesen Konzepten gut kompatibel. Allerdings wird sich im Folgenden zeigen, dass das ganzheitliche Herangehen eine drastische Umgewichtung zweier Faktoren nahelegt: Die von Michelle Craske und anderen als sehr wichtig und wirksam herausgestellte „Expectancy Violation“ tritt in den Hintergrund, ein von Craske auch untersuchter (Dour et al., 2016), aber zumeist nur am Rande erwähnter Faktor tritt dagegen in den Vordergrund: „positive Valence“.

Nach wie vor imponiert dabei sowohl das Feld der experimentellen Psychologie als auch die sich wesentlich von ihr ableitende Verhaltenstherapie (VT) als zersplittert in viele einzelne Ansätze, Methoden und Techniken. Oft wird der sich hieraus ergebende pragmatische Pluralismus zum Vorteil erklärt und in mancher Hinsicht mag das auch zutreffen. Aus Sicht des Autors überwiegen aufs Ganze gesehen allerdings deutlich die Nachteile.

So ist für die Entstehung von Angsterkrankungen dysfunktionales Lernen von zentraler Bedeutung, und auch hier zeigt sich diese Zersplitterung darin, dass die verschiedenen Lernformen in Theorie und Therapie isoliert voneinander behandelt werden. Tab. 1 zeigt eine sehr grundsätzliche Einteilung der Lernformen, 
Table 1. Hauptformen des Lernens

\begin{tabular}{|c|c|c|c|c|}
\hline Stufe & Psychische Inhalte & Prinzip & $\begin{array}{l}\text { Verinner- } \\
\text { lichung }\end{array}$ & Neuronale Prozesse \\
\hline $\begin{array}{l}\text { Einsichtslernen } \\
\text { (explizit) }\end{array}$ & $\begin{array}{l}\text { Begriffliches } \\
\text { Denken, Ein- } \\
\text { sicht (kognitive } \\
\text { Umstrukturierung) }\end{array}$ & $\begin{array}{l}\text { Verbindung- } \\
\text { durch Logik }\end{array}$ & $\begin{array}{l}\text { Diffe- } \\
\text { renzierte } \\
\text { Vernetzung }\end{array}$ & $\begin{array}{l}\text { Attraktorregulierte } \\
\text { Musterbildung in } \\
\text { Oszillator-Arrays, Ein- } \\
\text { sicht = Phasensprung }\end{array}$ \\
\hline $\begin{array}{l}\text { Konditionie- } \\
\text { rungslernen (im- } \\
\text { plizit u. explizit) }\end{array}$ & $\begin{array}{l}\text { „reflexhafte Mitak- } \\
\text { tivierung“, Einbin- } \\
\text { dung von Bildern }\end{array}$ & $\begin{array}{l}\text { Verbin- } \\
\text { dung durch } \\
\text { Koinzidenz }\end{array}$ & $\begin{array}{l}\text { Wieder- } \\
\text { holung, } \\
\text { emotionale } \\
\text { Intensität }\end{array}$ & $\begin{array}{l}\text { Hebb-Synapse, „elekt- } \\
\text { risches Schaltnetz“ }\end{array}$ \\
\hline $\begin{array}{l}\text { Habituations- } \\
\text { lernen (implizit u. } \\
\text { explizit) }\end{array}$ & $\begin{array}{l}\text { Abschwächung } \\
\text { oder Verstärkung v. } \\
\text { Reaktionen }\end{array}$ & $\begin{array}{l}\text { Abschwä- } \\
\text { chung od. } \\
\text { Verstärkung } \\
\text { v. vorbeste- } \\
\text { henden Ver- } \\
\text { bindungen }\end{array}$ & $\begin{array}{l}\text { Dauer v. } \\
\text { Reizung } \\
\text { bzw. } \\
\text { Reizpause }\end{array}$ & $\begin{array}{l}\text { Verringerung } \\
\text { bzw. Vermehrung } \\
\text { präsynaptischer } \\
\text { Transmitter- } \\
\text { Ausschüttungen }\end{array}$ \\
\hline
\end{tabular}

die an grundlegenden Arbeitsmodi des Gehirns orientiert ist und für praktische therapeutische Zwecke ausreichend und prägnant ist: Einsichtslernen, Konditionierungslernen und Habituationslernen. In den Lehrbüchern der Lernpsychologie werden diese (und weitere) Lernformen getrennt behandelt, oft mit Bezug auf besonders gut erforschte Modelle - Mensch, Ratte, Aplysia, entsprechend der obigen Reihenfolge. Gleiches gilt für die „Grundlagenteile" der VT-Lehrbücher, was sich dann auf den „Methodenteil“ überträgt - kognitive Verfahren, operante Verfahren, Konfrontationsverfahren u.a. werden separat voneinander gelehrt -, was auch im „Störungsteil“ durchgehalten wird: Bei der Behandlung einer bestimmten Störung erhalten die Verfahren getrennte Abschnitte. Teilweise wird sogar vor ihrer „Vermischung" gewarnt. So sollen etwa kognitive und konfrontative Verfahren nicht kombiniert angewandt werden. Menschliches Lernen, auch das Angstlernen, geht aber nicht auf in Einsicht, Konditionierung oder Gewöhnung allein. Therapien, die nur auf eine dieser Facetten zielen, können nur eingeschränkt effektiv sein. Menschliches Lernen ist immer ein ganzheitlicher Prozess, der auf verschiedenen Ebenen nach unterschiedlichen Prinzipien arbeitet, und dies parallel und integriert.

Tab. 1 bringt zu den o.g. Lernformen Erläuterungen und bezieht sie auf verschiedene Arbeitsmodi des Gehirns, die sich grundlegend unterscheiden, gleichwohl aber parallel prozessiert werden. Am verbreitetsten ist die als Grundlage des Konditionierungslernens relevante Vorstellung, dass das Gehirn grob gesagt wie ein durch Hebb-Synapsen verbundenes elektrisches Schaltnetz arbeitet. Als neuronale Grundlage für das Einsichtslernen taugt diese Vorstellung allerdings nicht. 
Gemessen an der kreativen, sprunghaft-fluiden Dynamik von Einsichtsprozessen ist sie zu träge-inkrementell.

Ähnliches gilt für zu „starre“ Vorstellungen im Zusammenhang mit der Computer-Metapher des etablierten Kognitivismus (Denken als algorithmische Informationsverarbeitung). Vielmehr ist von Folgendem auszugehen: Bei Prozessen des Einsichtslernens (und anderen komplexen, fluide-kreativen Verhaltensweisen) fungieren große Neuronenverbände als Arrays nichtlinear gekoppelter Oszillatoren, in denen selbstorganisierte, attraktorregulierte Musterbildungen stattfinden (dissipative bzw. synergetische Strukturen). Eine Einsicht wäre dann im Sinne eines Phasensprunges einer synergetischen Struktur zu verstehen. Für den Koordinationswechsel komplexer Motorbewegungen konnte der mathematisch-experimentelle Nachweis hierfür erbracht werden (Haken et al., 1985; Haken, 1996; Kelso, 1995). Diese Konzepte einer „synergetisch orientierten Psychologie/Hirnforschung“ sind gut kompatibel zu den aktuellen Entwicklungen im Bereich neuronale Netze und Deep Learning, die im Kern auf einer Simulation von Attraktordynamiken beruhen.

Für diese grundlagentheoretischen Aspekte muss auf die Literatur verwiesen werden (Hansch, 1988; Hansch, 1997; Hansch \& Haken, 2004; zusammenfassend: Hansch \& Haken, 2016; Haken \& Schiepek, 2010). Wichtige Vorläufer und Anknüpfungspunkte liegen im Bereich der Gestalt- und Ganzheitspsychologie (Stadler \& Kruse, 1986; Haken \& Stadler, 1990).

\section{Angsterkrankungen: Eskalation durch Teufelskreise und Chronifizierung durch dysfunktionales Lernen}

Zurück zur Therapiepraxis: Am fiktiven Beispiel der Herausbildung einer „Herzangst" aufgrund vermehrten „Herzstolperns" sei erläutert, wie man sich die Integration der in Tab. 1 unterschiedenen Lernformen vorstellen kann. Diese Vorstellung ist plausibel, der Selbsterfahrung zugänglich, durch klinische Beobachtungen gedeckt und gut kompatibel mit Experimentalbefunden und grundlegenden Konzepten aus Psychologie und Hirnforschung.

Den Ausgangspunkt bilden oft bestimmte, z.T. früh entstandene Dispositionen/ Vulnerabilitäten: eine besonders hohe Sensibilität für Körpersensationen z.B., oder prägende frühere Erfahrungen (z.B. der Herztod des Großvaters, dem über längere Zeit Herzrhythmusstörungen vorausgingen). Wegbereiter ist dann oft eine längere Phase mit chronischem Disstress, und manchmal lassen sich auch konkrete Auslöser dingfest machen - etwa ein reißerischer Fernsehbeitrag, in dem Dinge wie Notarzt, Herzrhythmusstörungen und Herzinfarkt vorkommen. Dadurch wird die Aufmerksamkeit auf das eigene Herz fokussiert und wenn der Betreffende Pech hat, ist er gerade in einer Phase, wo er - vielleicht gefördert durch den Stress - vermehrt harmlose Extrasystolen hat, die er nun als Herzstolpern 
wahrnimmt. Und nun beginnt - allein schon dadurch, dass er mehr darauf achtet der Teufelskreis „Angst vor der Angst“: das wahrgenommene Herzstolpern wird als Gefahr interpretiert, das erzeugt Angst, die damit verbundenen physiologischen Veränderungen intensivieren die Herzfunktion: Steigerung der Herzfrequenz, eventuell auch Vermehrung der Extrasystolen, das wiederum wird wahrgenommen, als Gefahrensteigerung interpretiert usw. Je mehr sich das Ganze zu einer Panikattacke auswächst, je mehr neue Symptome hinzutreten - Hyperventilation, Schwindel, womöglich thorakale Schmerzphänomene - desto katastrophisierender geraten die Gedanken: „Ich verliere die Kontrolle, ich falle um, das ist der Herzinfarkt, ich sterbe!“

Nachdem die Untersuchungen „ohne Befund“ und ohne Erklärung bleiben, drängt das Kohärenzbedürfnis zur Eigenrecherche - u.a. im Internet - und zur Konstruktion einer ängstigenden subjektiven Krankheitstheorie. In Abhängigkeit von vielen Patientenvariablen - Differenziertheit, medizinische Vorbildung, Neigung zu magischem Denken, Phantasie, Perfektionismus etc. - fallen diese Angsttheorien sehr unterschiedlich aus. Sie können dabei durchaus Elemente enthalten, deren plausible und sichere Widerlegung auch dem Fachmann nicht immer leichtfällt (z.B. passagere Koronarspasmen). Je länger der Betroffene Zeit hat, seine Angsttheorie "fachlich ungestört“ zu entwickeln, zu differenzieren und damit in seinen vorbestehenden kognitiven Strukturen zu vernetzen, desto stärker verinnerlicht sie sich und desto stärkere Angst wird durch dieses Negativ-Frame induziert.

Dies sind die Prozesse auf Stufe des Einsichtslernens - parallel und „unterhalb“ dieser Ebene laufen Prozesse der Konditionierung ab: auch ohne bewusste Aufmerksamkeit und eine Lernintention gibt es im Nervensystem auf basaleren Ebenen eine Tendenz zu verbinden, was gemeinsam aktiviert wird - und zwar umso mehr, je häufiger die gemeinsame Aktivierung erfolgt und je stärkere Emotionen daran beteiligt sind. Der Kernprozess ist in Abb. 1 oben gezeigt: Herzsensationen werden wahrgenommen, das aktiviert die Strukturen der Angsttheorie in ihrem jeweils aktuellen „Ausarbeitungsstadium“, die assoziativ mit negativen Bildern in Verbindung steht (Bilder aus eigener Erinnerung, aus den Medien oder Konstrukte der Vorstellung, bzw. eine Mischung aus diesen Quellen), dies nun induziert Angst, die je nach Situation mehr oder weniger stark im Teufelskreis eskaliert wird. Je öfter und emotional aufgeheizter dieser Kreis abläuft, desto stärker werden diese drei Elementgruppen bzw. ihre „neuronalen Repräsentationen“ synaptisch miteinander verbunden, so dass sie sich immer leichter, intensiver und ausgreifender wechselseitig aktivieren können. So wächst eine neuronale Netzwerkstruktur, die wir hier kurz als „Angstnetz“ bezeichnen (ein ähnliches Konstrukt wäre die „Furchtstruktur" nach Foa \& Kozak 1986). Wo immer dieses Angstnetz assoziativ berührt wird - immer startet es dann schnell als Ganzes hoch: Wenn das Gespräch auf Krankheits- oder Herzthemen kommt, wenn es im Körper ruckelt - etwa im 


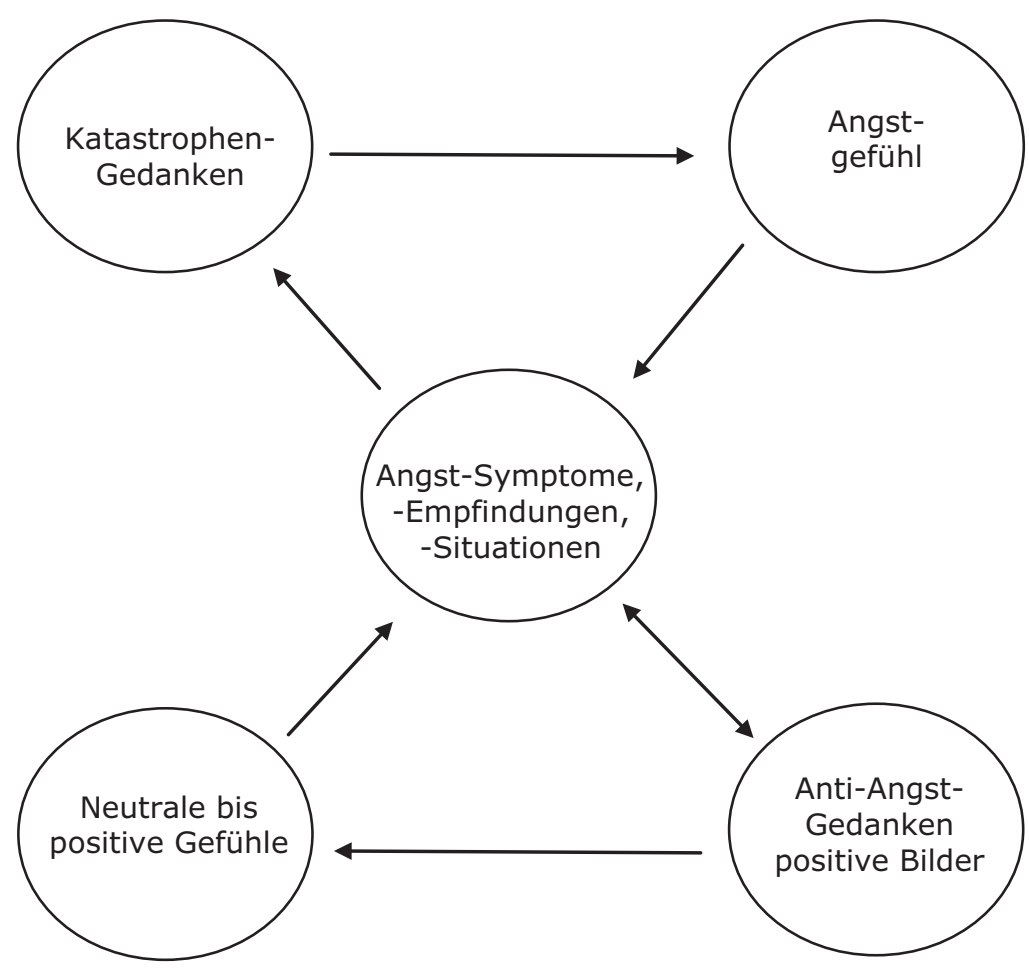

Abb. 1. Entstehung (oben) und „positives Überschreiben“ (unten) eines „Angstnetzes“.

Zug durch Schienenstöße-, wenn aus anderen Gründen Ängste entstehen. Und dann ist augenblicklich der gesamte Angstkomplex präsent: die Herzsensationen, die dahinterstehende Angsttheorie und starke Angst samt ihren sonstigen körperlichen Symptomen. Verschiedene Mechanismen können nun dazu führen, dass diese Ereignisse häufiger, heftiger werden, sich in einer bestimmten Richtung weiterentwickeln oder sich generalisieren. Dies wäre sozusagen eine horizontale Ausbreitung.

Doch auch in der Vertikalen "frisst" sich die Angst tiefer, indem sie die Stufe des Habituationslernens erreicht. Die Angst induziert Vermeidungsverhalten - in unserem Falle alles, was zu einer körperlichen Aktivierung führt: Zuerst entfällt der Sport, später womöglich auch das Treppensteigen, der Sex oder das KrimiGucken. Im Ergebnis steht ein Rückgang des Trainingsniveaus in Verbindung mit einer allgemeinen Sensitivierung, was auch zu einer immer intensiveren Reaktion auf die Stresshormone führt. Hierdurch werden die Herzsensationen verstärkt, auf der Einsichtsebene wird die Angst- und Krankheitstheorie bestätigt und verfestigt, der intensivierte Prozess intensiviert die Konditionierungen, und natürlich: es verstärkt sich das Vermeidungsverhalten. Ein ganzes Netzwerk von Teufelskreisen formiert sich. 
Es wird deutlich, wie sich alle drei Lernformen vernetzen: sie laufen gleichzeitig ab und stützen und verstärken sich gegenseitig. Gleichwohl gibt es Verursachungsgefüge und unterschiedliche Geschwindigkeiten.

Wie wohl bei den meisten menschlichen Ängsten spielen echte unkonditionierte Reize (also angeborene Angstauslöser) kaum eine primäre Rolle: Herzklopfen/ stolpern ist selbst emotional neutral und wird in der Lebenspraxis auch nicht unmittelbar mit einem starken Angstauslöser beantwortet (etwa mit einem Stromschlag wie im Labor). Das verursachende Hauptmomentum geht von dysfunktionalen Kognitionen aus (die assoziativ ggf. mit echten mehr oder weniger konkreten unkonditionierten Reizen zusammenhängen können, aber nicht müssen: Gedächtnisinhalte/-bilder im Zusammenhang mit Herzkrankheit/Herztod, etwa der sich in pectanginösem Schmerz windende Großvater). Die Formierung der Angsttheorie auf der Einsichtsebene ist also Hauptursache und geht am schnellsten. Die Ausbildung des Angstnetzes auf der Konditionierungsebene ist eine Folge, verläuft etwas langsamer, ist aber dann umso nachhaltiger. Das Vermeidungsverhalten bildet tendenziell das letzte Glied der Kette, müssen doch Sensitivierung und Angst erst eine gewisse Stärke erreicht haben, ehe auch schwer verzichtbare Aktivitäten im Alltag mit immer aufwendigeren Tricks umgangen werden. Damit schließt sich der „Angst-Kerker“ nun aber auch: Die Vermeidung verunmöglicht spontanes Korrekturlernen.

Wenn das Erlernen der Angst annähernd gemäß der hier aufgezeigten „PsychoLogik“ verläuft, dann gibt es gute Gründe für die Annahme, dass man das therapeutische Korrekturlernen am effektivsten gestaltet, wenn man es der gleichen Psycho-Logik folgen lässt.

\section{Angstbehandlung: Deeskalation und integriertes Korrekturlernen auf 3 Stufen}

Wie sähe eine Angstbehandlung aus, die dieser oben skizzierten Psycho-Logik folgt? Wie sähe eine idealtypische „therapeutische Rückabwicklung“ der o.g. Angstentwicklung aus?

Wiederum sei dies anhand des o.g. Herzangst-Beispiels aufgezeigt: Ersichtlich hätte man bei der Korrektur der inadäquaten subjektiven Krankheits- u. Angsttheorie anzusetzen. Man müsste dem Patienten helfen, sie zu explizieren und differenziert zu Papier zu bringen. Im nächsten Schritt hätte man psychoedukativ eine widerlegende Gegenposition zu erarbeiten und der Angsttheorie diese schriftlich gegenüberzustellen. Hierzu würden im vorliegenden Falle folgende Hauptelemente gehören - in Kurzform: Empfundenes Herzstolpern beruht auf harmlosen Extrasystolen, die in dieser Form bei den meisten gesunden Menschen auftreten. Biologische Prozesse sind von den uns aus dem Alltag modellgebend bekannten maschinellen Prozessen grundlegend verschieden: 
Unregelmäßigkeiten, Schwingungen und Schwankungen sind nicht nur keine Störung, sie sind ein unverzichtbares Positivmerkmal, sie sind essenziell für Flexibilität und Anpassungsfähigkeit organismischer Prozesse auf allen Ebenen. Gefahr droht eher, wenn diese Schwingungen verlorengehen, wenn also z.B. der Herzrhythmus seine Variabilität verliert: dies ist ein Zeichen von maximalem Stress und das Risiko für Herzprobleme steigt. Hinzu kommen etablierte Inhalte wie: Erklärung der Entstehung der Angst- und Paniksymptome aus den physiologischen Stress-Mechanismen im Zusammenwirken mit Teufelskreis-Mechanismen.

Diese „Anti-Angst-Theorie“ gilt es nun zu differenzieren und zu verinnerlichen, so dass sie zur ureigenen Überzeugung wird: umfangreich nachlesen auch $\mathrm{zu}$ angrenzenden Themengebieten, die neue Sichtweise in eigenen Worten aufschreiben, verbildlichen, aus verschiedenen Richtungen durchdisputieren, über sie „meditieren“, etc.

Im nächsten Schritt gilt es, für die angstbesetzten Hauptphänomene vor diesem neuen theoretischen Hintergrund eine griffige Deutung zu finden, die möglichst weit ins Positive geht, aber dennoch einen ausreichenden Realitäts- und Wissenschaftsbezug wahrt. Als positive Bilder haben sich hier z.B. bewährt: Ohne Spannung flattert das Seil im Wind, vor dem Zerreißen verharrt es in angespannter Starre. Oder: Der müde Boxer vor dem KO-Schlag steht am Fleck, der überlegene Boxer mit Reserven tänzelt unberechenbar. Kurzum: das Herz stolpert nicht, es tänzelt.

Auf dieser Basis gilt es nun Techniken aufzubauen, die es im Alltag ermöglichen, die Angst zu reduzieren und Teufelskreise zu unterbrechen:

Reframing: Bei Aufkommen von Angstmomenten im Alltag innerlich einen Schritt zurücktreten und bewusst die neue, Anti-Angst-Perspektive einnehmen: Herztänzeln und nicht Herzstolpern.

Achtsamkeit: Üben, den Angstmomenten in reiner Wahrnehmung ohne Interpretation und Wertung zu begegnen: Eigentlich fühlen sich Extrasystolen völlig neutral an, wenn ein Tier sie hätte, hätte es keinerlei Probleme damit. Das einzige Problem ist das (dem Menschen eigene) Negativdenken.

Akzeptanz: Vor diesem Hintergrund die ehemals angstbesetzten Phänomene positiv annehmen, sie nicht mehr „weghaben“ wollen.

Paradoxe Techniken: Noch einen Schritt weitergehen und sich die ehemals angstbesetzten Phänomene herbeiwünschen mit (schwarzem) Humor: „Na, liebes Herz, wie bist du heute drauf? Mach gern mal ein paar Freudenhüpfer, damit ich sehe, dass es dir gut geht. Und wenn du es schaffen solltest, während der Bahnfahrt zur Arbeit auf Puls 120 zu bleiben, würde mich das freuen, dann hätte 
ich gleich mein Herz-Kreislauf-Training weg und könnte mir das abendliche Joggen sparen!“

Diese Techniken bewirken bei Aufkommen der Angstmomente eine Reduktion der Angst im Hier und Jetzt. Dies ist eine Elementarerfahrung aus der Selbstbeobachtung. Zugleich gibt es Hinweise auf die neuronalen Mechanismen, mit denen der präfrontale Kortex dazu in der Lage ist, die Aktivität der Amygdala und die von ihr ausgelösten Reaktionen zu modulieren (Ochsner et al., 2002; Delgado et al., 2008). Es ist davon auszugehen, dass diese Techniken umso wirksamer sind, je weiter der oben besprochene Verinnerlichungsprozess des AntiAngst-Wissens fortgeschritten ist.

Gleichwohl: Zunächst wird er zumindest bei länger fortgeschrittenen Angststörungen lediglich zu einer geringen bis mäßigen Reduktion der Angst führen, denn: das Angstnetz auf der Stufe des Konditionierungslernens besteht ja noch fort. Seine Auflösung bzw. sein „inhibitorisches Überschreiben“ braucht mehr Zeit als die kognitive Umstrukturierung auf Stufe der Einsicht.

Aber auch dieser Prozess ist natürlich parallel zu den Einsichtsprozessen schon im Gang, sein Fortschreiten hängt ab von der Zahl der Wiederholungen, mit der die o.g. Techniken ausgeführt werden. Er ist im unteren Teil von Abb. 1 dargestellt: Die noch angstintegrierten Momente werden in einen neuen, positiven Interpretationskontext gestellt, dort zunehmend assoziativ integriert und durch Wiederholung und neue, zunehmend positive emotionale Erfahrungen immer fester und differenzierter ins Positive „einkonditioniert“.

Aufgrund der zeitlichen Trägheit dieser Um- bzw. Neukonditionierungen insbesondere bei schweren, chronifizierten Angsterkrankungen macht es Sinn, diese Vorgänge gezielt zu beschleunigen. Dies ist u.a. möglich durch spezielle Imaginationsübungen folgender Art. Anknüpfend an die kognitive Vorarbeit wird ein autoinstruktiver Text erstellt, der wichtiges Material der Anti-Angst-Theorie in griffigen Formeln auf den Punkt bringt, mit Bildern verbindet, die positiv besetzt sind und sich womöglich auf frühere Kompetenzerfahrungen des Betroffenen bezieht. Um im gewählten Beispielkontext zu bleiben: Ein Betroffener, der früher einmal ein guter Boxer war, könnte sich vorstellen, sein Herz sei ein unüberwindlich fitter „innerer Boxer", der gelegentlich vor lauter Übermut Doubletten gegen die Thoraxwand schlägt („weiblichere“ Alternativen: die „innere Tennisqueen", die Bälle gegen die Thoraxwand schlägt, ein herumtollendes Fohlen). Der nächste Schritt wäre, diesen Text auswendig zu lernen. Man könnte ihn auch gesprochen aufnehmen, um ihn in Endlosschleife abspielbar zu machen. In den Imaginationsübungen besteht die Aufgabe nun darin, sich diese Inhalte präsent zu machen - sie aufsagen oder abspielen -, sich die angstbesetzten Phänomene vorzustellen, beides intensiv innerlich miteinander in Verbindung zu bringen, die Phänomene gewissermaßen neu und positiv kognitiv „einzukleiden“, so dass 
neutrale bis positive Gefühle entstehen, sich sukzessive in eine positive Stimmung „hineinzupushen“, in der diese mentale Neukonstellation - (Angst-)Momente, positive Neuinterpretation und positive Gefühle - immer fester konditionierend „verbacken“ wird. So entsteht zum Angstnetz ein konkurrierendes „Positivnetz“, das sich ausbreitend erstarkt, bei Manifestation der (Angst-)Momente zunehmend die Aktivität an sich zieht und zugleich vom alten Angstnetz abzieht. Letzteres wird zunehmend gehemmt, stillgelegt, schrumpft und löst sich manchmal vielleicht sogar weitgehend auf (genauere Beschreibung des Procederes in Hansch, 2017). (Grundsätzlich besteht die Möglichkeit, die positiven Anti-Angst-Inhalte in weiteren Modi wie Musik und Bewegung zu verankern, wobei der Ansatz des Embodiment berührt wird: Bisher zwei der vom Autor behandelten Patienten machten aus ihrem Anti-Angst-Text einen vertonten Rap-Song und tanzten dazu, der eine im angehaltenen Klinik-Fahrstuhl, der andere ein einem leeren Zugabteil.)

In einem damit wird ersichtlich schon der nächste anstehende Schritt in Teilen vorweggenommen: die Exposition auf der Stufe des Habituationslernens. Wenn man sich im Rahmen der o.g. Imaginationsübungen das Herzstolpern/-tänzeln oder andere Angstmomente/-situationen vorstellt, ist das eine Konfrontation in sensu. Das erleichtert den Übergang zur Expositionstherapie in vivo, bereitet ihn vor und setzt den bisherigen Prozess bruchlos fort.

Bei dieser Exposition in vivo geht es nun darum, die Angstmomente/-situationen in angemessenen Steigerungsschritten aufzusuchen/zu erzeugen und sich ihnen zu stellen - in der Psycho-Logik des hier vertretenen Ansatzes mit folgenden Zielen:

1. Anstreben und üben, das zuvor kognitiv-imaginativ aufgebaute Anti-Angst-Mindset möglichst auch in der Realsituation innerlich stabil zu halten bzw. es immer wieder zu aktivieren, so dass möglichst wenig Angst aufkommt bzw. möglichst viel von der mit der Neuinterpretation verbundenen positiven Emotionalität erhalten bleibt bzw. geweckt wird. Im gewählten Beispiel-Fall könnten die Expositionsschritte wie folgt aussehen: Kniebeugen machen, Treppensteigen, Joggen, Sprinten mit der Absicht möglichst zunehmend starke und spürbare Herzaktivität zu erzeugen; üben, diese im neuen Interpretationsgewand freudvoll zu erleben: „Mein Herz ist gesund, es spurtet wie ein Olympiaboxer beim Training, der gelegentlich mal ein paar tänzelnde Schattenbox-Einlagen macht!“ Oder: „Es ist wie ein junges Fohlen, das Freudenhüpfer macht!"

2. Gegebenenfalls: Die Begleit-/Restangst und deren Symptome adäquat kognitiv einordnen („reframen“) und mit innerer Distanz „aushalten“ lernen. Üben, trotz und mit leichten bis mittleren Stress- und Angstsymptomen handlungsfähig zu bleiben und seine objektiven, positiven Ziele zu erreichen.

3. Vorher objektive Ziele definieren und diese möglichst unabhängig vom Befinden anstreben und erreichen. Diese Zielerreichung ist das „Erfolgs- und Abbruchkriterium“ für die Exposition. Im gewählten Beispiel-Fall: z.B. 50 Kniebeugen, 3 km Joggen etc. 
4. Korrekturlernen - Erfahrungen machen wie die folgenden: Die befürchteten Katastrophen treten nicht ein; ich kann mich auf mich und meinen Körper ausreichend verlassen; die Angst nimmt nicht immer weiter zu; ich bleibe auch mit stärkerer Angst handlungsfähig; nach einiger Zeit sinkt das Angstniveau von allein deutlich. Zugleich wird der Gesamtprozess über alle Lernstufen fortgesetzt und abgeschlossen: die Anti-AngstTheorie wird bestätigt und verfestigt, die positive Umkonditionierung fortgesetzt und beschleunigt, es finden Gewöhnung- und Abhärtungsprozesse statt, es werden Bewältigungskompetenzen gestärkt, es kann eine Aufwärtsspirale in Gang kommen.

Das Kernstück der Behandlung ausgeprägter Angststörungen - die Expositionstherapie - wird so zu einem ganzheitlichen Prozess, in dem über drei Stufen ein kohärentes Anti-Angst-Mindset aufgebaut wird (Abb. 2). Dabei verankern und stabilisieren sich alle Inhalte auf allen Stufen in allen Modi wechselseitig (die Einsichtsebene stellt positive Frames zur Verfügung, die bei Imaginationen und Situationswahrnehmung immer intuitiv „mitschwingen“, die Expositionssituationen können imaginativ vorweggenommen werden, etc.).

\section{Exposition als Schlusspunkt ganzheitlichen Korrekturlernens}

Wodurch unterscheidet sich die Expositionstherapie in diesem ganzheitlichen Kontext von der „etablierten“ Expositionstherapie? Vor allem in drei Punkten:

\section{Verhältnis Kognition - Exposition}

Wie eingangs schon erläutert, steht die Expositionstherapie in der herkömmlichen Form zumeist isoliert für sich. Zwar wird eine „kognitive Vorbereitung“ empfohlen, diese bezieht sich aber auf das Rational der Expositionstherapie an sich und nicht auf ein spezifisches Reframing der Angstinhalte, wie im hier vorgeschlagenen Verfahren. Oft wird explizit von einer „Vermischung“ kognitiver und konfrontativer Verfahren abgeraten, weil sich - unter Bezugnahme auf bestimmte Befunde aus Tierversuchen -

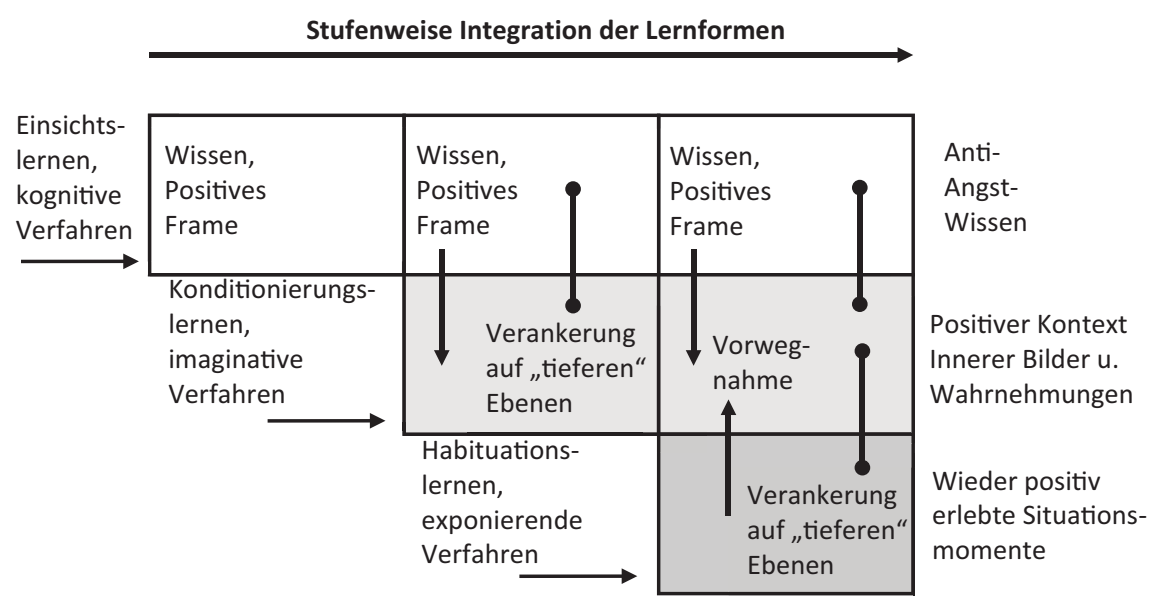

Abb. 2. Sukzessiver Aufbau eines kohärenten Anti-Angst-Mindset über die drei Lernstufen. 
beide Prozesse stören könnten (zusf. Diskussion s. LeDoux, 2015, S. 447ff.). Es bleibt fraglich, wie weit man diese Befunde überhaupt auf den Menschen übertragen kann. Was aber sicher stimmt: Wollte man alles in eine Sitzung packen und den Patienten 15 Minuten vor Konfrontationsbeginn mit den Inhalten des Reframing vertraut machen, käme es zu einer Überforderung. Im hier vorgeschlagenen Verfahren geschieht dies aber nicht: Die neuen kognitiven Inhalte werden im Vorfeld systematisch aufgebaut und verinnerlicht, so dass sie zu Expositionsbeginn stabil zur Verfügung stehen. Das Entscheidende Momentum der hier vorgeschlagenen Form von Expositionstherapie ist es gerade, das neu aufgebaute positive Anti-Angst-Frame gegenüber dem noch hochdrängenden alten Angst-Frame stabil zu halten. Es handelt sich im Kern um eine "New-Frame-Stabilizing-Therapy“.

Ganz abgesehen davon, dass all dies im Grunde nur vernünftig ist und einer Systematisierung alltäglicher und evidenter Lebenserfahrungen entspricht - es gibt interessanterweise durchaus auch Experimentalbefunde, die dieses Herangehen stützen. So zeigte sich in einer Studie unter Beteiligung von Craske, dass die Exposition bei Spinnenphobie wirksamer und nachhaltiger ist, wenn man Spinnen zuvor durch kognitive Umstrukturierung mit einer möglichst positiven Valenz ausstattet (die Probanden hörten vor der Exposition eine positive Märchengeschichte über eine fleißige, für andere Tiere hilfreiche Spinne: Charlottes Web von E.B. White, s. Dour et al., 2016).Offenbar ist Craske dieser Spur aber nicht weiter gefolgt - vielmehr wählt sie die entgegengesetzte Richtung: In ihren zusammenfassenden Empfehlungen zur Expositionstherapie (Craske et al., 2014) streicht sie heraus, dass die Effektivität des Korrekturlernens mit der Stärke des Überraschungseffektes wächst, der aus möglichst krasser Verletzung vorheriger Erwartungen resultiert. Danach wäre auf positive kognitive Umstrukturierungen im Vorfeld zu verzichten und vielmehr die negative Valenz der Angstmomente explizit herauszuschärfen. In o.g. Beispiel müsste das dann in etwa so aussehen: „Wenn wir jetzt Sprinten gehen - was ist ihre schlimmstmögliche Befürchtung? Dass Sie einen Herzinfarkt erleiden. Bei welcher Belastung fürchten Sie, dass das mit Sicherheit eintreten müsste? Ok, nach einem maximalen 50-MeterSprint.“ Mit ernstem Blick auf das Notfalltelefon: „Gut, dann lassen Sie uns das jetzt testen - die Strecke ist abgesteckt, sprinten Sie los! Sie wissen, wir sind in Nähe der Notaufnahme." Die meisten Betroffenen würden in dieser Situation sicher alles andere tun, nur nicht mit wirklich maximaler Kraft sprinten.

Im Rahmen des hier vorgeschlagenen Ansatzes wird davon ausgegangen, dass der kognitiv-imaginative Aufbau eines stabilen, positiven Anti-Angst-Mindset es dem Betroffenen überhaupt erst ermöglicht, sich auf eine wirklich maximale Exposition einzulassen. Gleichwohl, die Explikation früherer Erwartungen und das Bewusstmachen ihres Widerlegtseins mit dem Ziel einer maximalen Diskrepanz ist sinnvoll und dürfte auch in dieser Form einen Effekt haben: „Super, ich sehe, ihnen schlägt nach diesem Sprint wirklich das Herz zum Halse heraus. Im vorher von uns aufgebauten Vertrauen auf ihren Körper haben sie es jetzt wirklich geschafft, sich einmal bis an die Grenzen zu belasten! Denken sie einmal zurück: Wenn ich ihnen vor 3 Monaten gesagt hätte, dass sie das tun würden, dass sie Puls 180 aushalten - was hätten sie damals befürchtet, das geschehen würde? Sie wären sicher gewesen, einen Infarkt zu bekommen. Sie sehen, nichts dergleichen ist auch nur im Entferntesten eingetreten. Stattdessen fühlen sie sich pudelwohl! Speichern sie diese Erfahrung gut ab!“ 
2. Wie wichtig ist es, dass es zur Aktivierung starker Angst kommt?

In vielen Beschreibungen/Empfehlungen der etablierten Expositionstherapie wird gefordert, dass man die Bedingungen so herzustellen hätte, dass auch wirklich starke Angst aufkommt. Nur dann könne man ja auch das Ziel erreichen - das Erleben der Abnahme der Angst. Das leuchtet allenfalls ein, wenn das Problem fast ausschließlich die „Angst vor der Angst“ ist bzw. Unvermögen oder Unwille, negative Gefühle wie stärkere Angst zuzulassen und auszuhalten. So umschrieben ist das aber nur selten der Fall. Zumeist bezieht sich die Angst ja primär auf etwas anderes: z.B. auf die als Krankheitszeichen gedeuteten körperlichen Angstsymptome wie Herzrasen, Schwitzen oder Schwindel - die kann man aber auch anderswo und normalerweise völlig angstfrei erleben (beim Sprinten, in der Sauna oder auf dem Drehstuhl); auf bestimmte öffentliche Situationen (Busfahren, Vorträge halten) oder auf den inneren Umgang mit existenziellen Risiken (sozialer Abstieg, Vereinsamung, Krankheit, Sterben) - und auch mit all diesen Gegebenheiten wäre ein weitgehend angstfreier Umgang normal und anzustreben. Wenn es dem Patienten durch intensive Vorbereitung in Form des kognitiv-imaginativen Aufbaus eines Anti-Angst-Mindset gelingt, sich bei der Exposition diesen Gegebenheiten wirklich und ohne Ablenkung zu stellen und dabei durch stabiles Reframing schon gar keine inadäquate Angst mehr aufkommt, dann sind wir doch auf dem Weg zum Ziel! Wozu denn dann noch künstlich Ängste schüren? Es gilt, sich an die genannten primären Angstauslöser zu gewöhnen - wenn dies ohne Angst gelingt, ist ja auch die Angst vor der Angst kein Thema mehr.

Hat man - v.a. im Rahmen der etablierten, „isolationistischen“ Herangehensweise -die Neigung, die Expositionstherapie für sich zu betrachten, wünscht man sich vielleicht einen „knalligen Effekt“ in Form einer grundstürzenden Einsicht oder eines spektakulären Angstabfalls. Sieht man die Exposition aber als Schlusspunkt einer ganzheitlich-integrierten Behandlung mit dem Patienten im Fokus, der wieder handlungsfähig werden soll mit möglichst wenig Angst - dann braucht man all das nicht mehr und ist froh darum.

Es leuchtet nicht ein, sich besonders starke positive Wirkungen dadurch zu erkaufen, dass man die Dinge zuvor besonders weit ins Negative bewegt. Es ist nicht vertretbar, inadäquate Kognitionen oder Emotionen beim Patienten therapeutisch zu verstärken (quasi in Analogie zu folgendem, bekannten Scherz: „Guter Mann, warum bloß schlagen Sie sich immer mit dem Hammer auf den Daumen?“ „Es ist so schön, wenn der Schmerz nachlässt!").

3. Wie wichtig ist es, dass die Angst während der Therapie verschwindet?

In der klassischen Expositionstherapie wird großer Wert auf die Abnahme des Angstpegels gelegt, eine weitgehende Abnahme der Angst wird als Erfolgskriterium definiert. Wie weiter unten noch deutlicher werden wird, liegt es in der Logik des hier vertretenen Ansatzes, den Fokus der Aufmerksamkeit mehr von Gefühlen, Befinden und Innerlichkeit weg und hin auf das nach außen gewandte Tun zu richten. Gefühle unterliegen nicht unserer direkten Willenskontrolle, sie lassen sich nur indirekt über eine entsprechende Steuerung von Denken und äußerem Verhalten beeinflussen. Jeder Versuch, Gefühle kurzschlüssig direkt beeinflussen zu wollen, birgt die Gefahr infolge von Teufelskreismechanismen konträre Effekte zu erzielen. Funktionale Gefühle sind immer Begleitresultat eines auf sinnvolle und positive 
Ziele gerichteten inneren oder äußeren Tuns. Im Sinne unserer Verfahrensintegration zeigen sich an dieser Stelle auch die Verfahren der 3. Welle der VT, insbesondere die Akzeptanz-Commitment-Therapie sehr gut anschlussfähig. Das gleiche gilt für die diesbezüglichen Aspekte im Umfeld der Konzepte des Inhibitionslernens, wo der Fokus ja auch auf dem Erlernen von Bewältigungskompetenzen liegt. Wie unten deutlich werden wird, verfolgt der hier vertretende Gesamtansatz etwa gleichgewichtig die folgenden drei Strategien: Angstreduktion, Akzeptanz der Angst, Stärkung der Bewältigungskompetenzen im Verfolgen positiver Ziele. Wie oben ausgeführt, wird als das wichtigste Erfolgskriterium bei der Exposition die Erreichung vorher festgelegter objektiver Ziele definiert.

Man könnte sich fragen: klingt nicht „Expositions-/Konfrontatiostherapie“ überhaupt etwas zu passiv-rezeptiv in diesem neuen Kontext? Wäre ein Begriff wie „therapeutisches Expositionshandeln“ nicht passender? In einer zusammenfassenden Definition: Therapeutisches, auf positive Ziele gerichtetes Expositionshandeln unter „Wegreframen“ von soviel Angst wie möglich und Akzeptanz der „Restangst" in der Hoffnung, dass sich auch diese „Restangst“ im Laufe der Zeit noch vermindert durch: Gewöhnung, durch das Gefühl von Stärkegewinn infolge Kompetenzwachstum, durch eine Verbesserung der Lebensbalance insgesamt (Stressreduktion, Ressourcenaktivierung).

\section{Das ganze Bild im 2-Ebenen-3-Stufen-Schema}

Damit ist der Kernprozess einer Integration spezifischer Verfahren der Angstbehandlung besprochen. Mit großer Wahrscheinlichkeit - so die hier vertretene Grundidee - sind an der Entwicklung von Angststörungen die folgenden Momente in unterschiedlicher Ausprägung und tendenziell in folgender Reihenfolge beteiligt: Dispositionen, Wegbereiter (chronischer Disstress), Auslöser, Eskalation durch Teufelskreismechanismen, Chronifzierung durch dysfunktionales Lernen auf den drei Stufen Einsicht, Konditionierung, Habituation. Bis jetzt ist die „Rückabwicklung" dieses Prozesses gemäß seiner eigenen Entwicklungslogik bezüglich der Momente Auslöser, Eskalation und Chronifizierung besprochen.

Zum ganzen Bild gehört, auch die Momente Wegbereiter und Dispositionen zu bearbeiten, wo nun auch unspezifische Herangehensweisen integriert werden können.

Zum Erfassen dieses ganzen Bildes wurde das in Abb. 3 in der einfachsten Version gezeigte 2-Ebenen-3-Stufen-Schema (kurz 2e3s-Schema) entwickelt, das in verschiedenen Abwandlungen auch für die Psychoedukation genutzt wird es ist gut kompatibel zu jüngeren ganzheitlichen Modellbildungen, etwa bei Mischel (2015) oder Kahneman (2012). Der untere Teil, die Ebene 1, wird vom Selbst gebildet: der „unbewusste Bereich“ von Gehirn und Psyche. Hier haben die Gehirnzentren ihren Sitz, die für die basalen Lebensfunktionen und auch die Grundgefühle zuständig sind, u.a. für Furcht/Angst (im Bereich limbisches System, Amygdala etc.). Getriggert durch äußere oder innere Auslöser entsteht hier die Angst samt der spontanen Verhaltenstendenzen (Erstarrung, Flucht, Kampf) 
und den bekannten Körperreaktionen („Stressreaktion“). Dies wird nun auf Ebene 2, den bewusstseinsfähigen Gehirnbereichen (insbesondere Frontalhirn), wahrgenommen und es erfolgt eine zweite, bewusste Reaktion auf das Primärgeschehen. Dies ist die kritische Stelle, wo durch Fehler die Teufelskreise entstehen und krankhafte Angst eskaliert (Katastrophisieren, Angst vor der Angst, Kampf gegen die Angst und ihre Symptome).

Die Therapie hat nun differenziert an beiden Ebenen anzusetzen: Auf Ebene 1 geht es sozusagen um eine „Beruhigung“ des Selbst mit dem Ziel Auftretenswahrscheinlichkeit und Intensität pathologischer Ängste zu vermindern. Wesentlich wird dies erreicht, durch unspezifische, nicht erläuterungsbedürftige Therapiemaßnahmen, wie sie auf der linken Seite von Abb. 3 aufgeführt sind. Auf Ebene

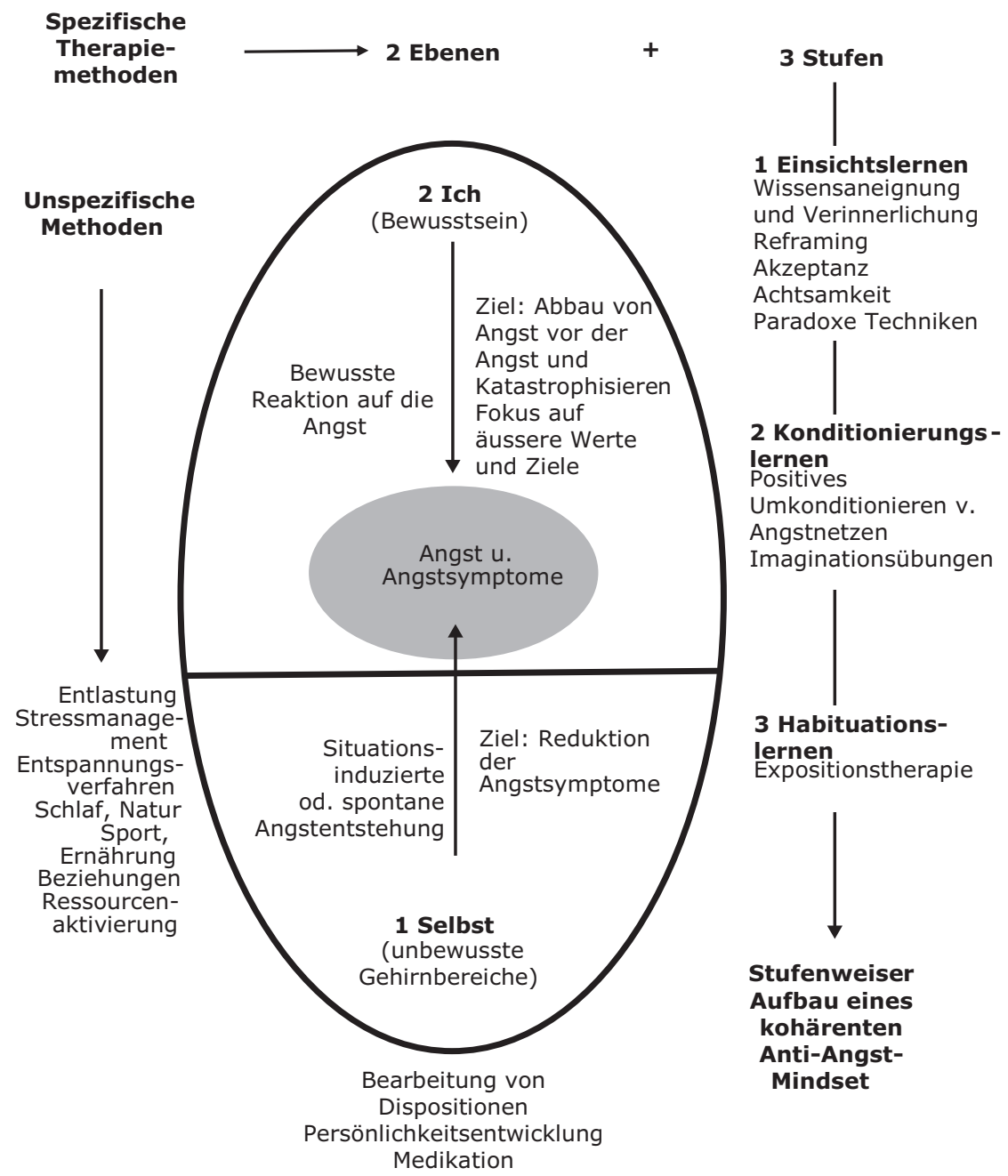

Abb. 3. Angstbehandlung im Gesamtüberblick: Das 2e3s-Schema. 
2 bestehen die folgenden Ziele: Abbau von Katastrophisieren und Angst vor der Angst, Vergleichgültigung der „Restangst“, Refokussierung auf positive äußere Ziele. Diese Ziele werden überwiegend erreicht durch die über drei Stufen integrierten, kognitiv-imaginativ-konfrontativen Therapieverfahren, die eben erläutert wurden. Schließlich bleibt der untere Pol: Bearbeitung von Dispositionen Traumata, frühe Prägungen, Persönlichkeitsakzentuierungen, dysfunktionale Reaktions- und Verhaltensmuster -, wobei auch gestalttherapeutische, tiefenpsychologisch fundierte und andere Verfahren anschlussfähig wären. Und last but not least: Persönlichkeitsentwicklung. Im salutogenetischen Sinne sollten sich der Erwerb von Selbstkompetenz und der Aufbau von Ressourcen bessernd auf Angsterkrankungen und andere psychische Störungen auswirken.

Dieses 2e3s-Schema bietet eine prägnante Systematik, die den therapeutischen Blick auf alle relevanten Optionen lenkt. In dem ausführlichen Selbsthilfebuch/Therapiebegleiter „Angst selbst bewältigen“ werden die Möglichkeiten der (Selbst-)Behandlung für die wichtigsten Angsterkrankungen nach diesem Schema bearbeitet (Hansch, 2017).

\section{Abstracts}

Nicht zu Unrecht gilt die Behandlung von Angsterkrankungen als die Paradedisziplin der Verhaltenstherapie (VT). Hier wie auch generell zeigt sich die VT dabei aber als zersplittert in eine Vielzahl von Einzelmethoden: Verschiedene Lernformen - Einsichtslernen, Konditionierungslernen und Habituationslernen - werden in ihrem Beitrag zu Angsterkrankungen isoliert voneinander konzipiert. Entsprechend stehen auch auf diesen Lernformen basierende Therapiemethoden für sich. Dadurch werden wichtige Synergiepotenziale verschenkt. Menschliches Lernen, auch und gerade das Lernen und Verlernen von pathologischer Angst, ist aber immer ein ganzheitlicher Prozess. Der Artikel skizziert eine ganzheitliche Psycho-Logik der Eskalation und Chronifizierung pathologischer Angst unter integrierendem Einbezug der o.g. Lernformen. Hieraus leitet sich eine verfahrensintegrierende VT ab, die die etablierten Behandlungsmethoden so kombiniert, dass Synergiegewinne entstehen, was an einem Fallbeispiel verdeutlicht wird. Den theoretischen Hintergrund bildet die Theorie der Selbstorganisation komplexer Systeme, insbesondere die Synergetik - ein Feld, in dem wichtige Aspekte der Gestalttheorie aufgehoben sind. It is not without reason that the treatment of anxiety disorders is considered the showpiece of behavioral therapy (BT). Here as well as in general, however, the BT shows itself fragmented into a multitude of individual methods: Different forms of learning - insight learning, conditioning learning and habituation learning - are designed in isolation from each other in their contribution to anxiety disorders. Correspondingly, treatment methods based on these forms of learning stand for themselves. This gives away important synergy potential. Human learning, also and especially learning and unlearning of pathological anxiety, is always a holistic process. The paper outlines a holistic psycho-logic of the escalation and chronification of pathological anxiety, integrating the above mentioned forms of learning. This leads to the derivation of a method-integrating BT, which combines the established treatment methods in such a way that synergy gains are achieved, as 
illustrated by a case study. The theoretical background is formed by the theory of the selforganization of complex systems, in particular synergetics - a field in which important aspects of gestalt theory are implied.

Schlüsselwörter: Verhaltenstherapie, Angsterkrankungen, Ganzheitlichkeit, Verfahrensintegration.

\section{Literatur}

Craske, M. G., Treanor, M., Conway, C. C., Zbozinek, T. \& Vervliet, B. (2014). Maximizing exposure therapy: an inhibitory learning approach. Behaviour, Research and Therapy, 58, 10-23.

Delgado, M. R., Nearing, K. I., LeDoux, J. E. \& Phelps, E. A. (2008). Neural Circuitry Underlying the Regulation of Conditioned Fear and its Relation to Extinction. Neuron, 59, 829-838.

Dour, H. J., Brown, L. A. \& Craske, M. G. (2016). Positive valence reduces susceptibility to return of fear and enhances approach behavior. Journal of Behavior Therapy and Experimental Psychiatry, 50, 277-282.

Foa, E. B. \& Kozak, M. (1986). Emotional Processing of Fear: Exposure corrective Information. Psychological Bulletin, 99, 20-35.

Haken, H. (1996). Principles of Brain Functioning. A synergetic Approach to Brain Activity, Behavior and Cognition. Berlin, Heidelberg: Springer Verlag.

Haken, H. \& Stadler, M. (1990). Synergetics of Cognition. Berlin, Heidelberg: Springer Verlag.

Haken, H., Kelso J. A. S. \& Bunz, H. (1985). A theoretical model of phase transitions in human hand movements. Biological Cybernetics, 51, 347-356.

Haken, H. \& Schiepek, G. (2010). Synergetik in der Psychologie. Göttingen: Hogrefe.

Hansch, D. (1988). Psychosynergetik - Neue Perspektiven für die Neuropsychologie? Grundriss einer psychosynergetischen Theorie emotionaler und motivationaler Prozesse. Zeitschrift für Psychologie, 196, 421-436.

Hansch, D. (1997). Psychosynergetik. Die fraktale Evolution des Psychischen. Opladen: Westdeutscher Verlag. Hansch, D. (2017). Angst selbst bewältigen. München: Knaur.

Hansch, D. \& Haken, H. (2004). Zur theoretischen Fundierung einer integrativen und salutogenetisch orientierten Psychosomatik. Gestalt Theory, 26 (1), 7-34.

Hansch, D. \& Haken, H. (2016). Synergetik in Hirnforschung, Psychologie und Psychotherapie. In: T. Petzer \& S. Steiner (Hrsg.), Synergie. Kultur und Wissensgeschichte einer Denkfigur. (S. 365-388). Paderborn: Fink.

Hoyer, J. \& Heinig, I. (2015). Wie sind Angststörungen verhaltenstherapeutisch zu behandeln? Neue Entwicklungen. Psychotherapie im Dialog, 2, 16-21.

Kahneman, D. (2012). Schnelles Denken, langsames Denken. München: Siedler.

Kelso, J. A. S. (1995). Dynamic Patterns. The Self-Organization of Brain and Behavior. Cambridge: MIT Press. LeDoux, J. (2015). Angst. Wals: Ecowin.

Mischel, W. (2015). Der Marshmallow-Effekt. München: Pantheon.

Ochsner, K. N., Bunge, S. A., Gross, J.J. \& Gabrieli, J. D. E. (2002). Rethinking Feelings: An fMRI Study of the Cognitive Regulation of Emotion. Journal of Cognitive Neuroscience, 14 (8), 1215-1229.

Stadler, M. \& Kruse, P. (1986). Gestalttheorie und Theorie der Selbstorganisation. Gestalt Theory, 8, 75-98.

Dr. med. Dietmar Hansch, Jg. 1961, Studium Humanmedizin, Physik und Philosophie in Berlin und Hagen. Facharzt Innere Medizin mit Zusatztitel Psychotherapie (Schwerpunkt Verhaltenstherapie). Nach klinischer und wissenschaftlicher Tätigkeit im Bereich Psychosomatik/Psychotherapie (UK Charitè, UK Aachen) seit 2014 Leiter Schwerpunkt Angsterkrankungen an der Privatklinik Hohenegg (Meilen bei Zürich). Versuch ganzheitlicher Modellbildungen für die psychische Veränderung durch Integration u.a. folgender Forschungsfelder: Selbstorganisation/Synergetik, Gestaltpsychologie/-theorie, Evolutionspsychologie, Verhaltenstherapie. 11 Bücher.

Address: Dr. Dietmar Hansch, Privatklinik Hohenegg, Hohenegg 1, Postfach 555, CH-8706 Meilen

E-mail: dietmar.hansch@hohenegg.ch

Orcid: 0000-0002-6608-9533 